\title{
General overview of recent results from the Pierre Auger Observatory
}

\author{
R. Caruso on behalf of the Pierre Auger Collaboration ${ }^{1,2, *}$ \\ ${ }^{1}$ Department of Physics and Astronomy, University of Catania and INFN-Catania, Via S. Sofia 64, 95123 Catania, Italy \\ ${ }^{2}$ Observatorio Pierre Auger, Avenida San Martin Norte 304,5613 Malargue, Mendoza, Argentina \\ *Full author list: http://www.auger.org/archive/authors \protect_2010 \protect_11.html
}

Received: 14 November 2010 - Revised: 21 February 2011 - Accepted: 3 March 2011 - Published: 19 October 2011

\begin{abstract}
Since June 2008 the Pierre Auger Observatory, designed for the research of ultra high energy cosmic rays, is taking data in its final configuration. In this paper I will present a short overview of its recent scientific results. More specifically, I will discuss the all particle energy spectrum and the evidence for the GZK features, the measurement of the elongation rate and the resulting inferences on mass composition, the evidence for anisotropy in the subset of the highest energy events.
\end{abstract}

\section{Introduction}

Cosmic rays are energetic particles and nuclei from space that strike Earth's atmosphere in all directions. Their energies vary from $10^{8} \mathrm{eV}$ to $10^{20} \mathrm{eV}$. The region of our interest is for energies beyond $10^{19} \mathrm{eV}$ where their flux decreases very rapidly with energy up to less than 1 particle $/ \mathrm{km}^{2}$ per century. They are so rare that in the last fifty years only a handful of 100- EeV particles have been detected from different experiments with different techiniques (Nagano and Watson, 2000). The identification of the sources, the characteristic features of the spectrum and the real nature of the ultra high energy cosmic rays (UHECRs) have been a great challenge since their first observation in 1962 (Linsley, 1963) and after decades of efforts, open questions are still present. Simply stated, we don't know what they are, where they come from or how they got here from there.

\section{The Pierre Auger Observatory}

Construction of the first stage of the Pierre Auger Observatory began in 2001 with a prototype system. It has been col-

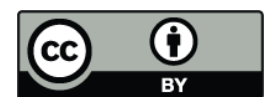

\section{Correspondence to: R. Caruso}

(rossella.caruso@ct.infn.it)

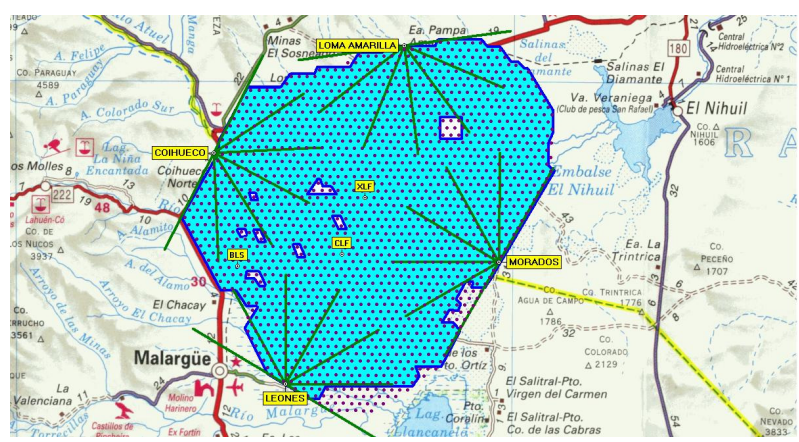

Fig. 1. Layout of the Pierre Auger Observatory as of 27 April 2010. The dots represent the position of each Cherenkov station, the yellow labels represent the four sites each grouping six fluorescence telescopes with green lines indicating their field of view .

lecting data since early 2004 and it was completed in 2008. Today more than 280 physicists from more than 70 institutions in 17 participating countries around the world are collaborating. It is the first experiment in cosmic ray physics characterized by very high performances using the hybrid technique (see Sect. 3).

The Pierre Auger Observatory (PAO) (Abraham et al., 2004) is located near Malargue, a town in Mendoza province over a vast plain of $3000 \mathrm{~km}^{2}$ known as the Pampa Amarilla (yellow prairie) in western Argentina near the Andes Mountains. This location was selected due to the low population density $\left(<0.1 \mathrm{~km}^{-2}\right)$, special climate (dry and clear), low pollution and flatness of landscape. The Observatory is placed at $35.2^{\circ} \mathrm{S}$ latitude and $69.5^{\circ} \mathrm{W}$ longitude at $1400 \mathrm{~m}$ above sea level corresponding to the atmospheric depth of $X=880 \mathrm{~g} \mathrm{~cm}^{-2}$. Figure 1 shows the layout of the PAO as of 27 April 2010 with its 1600 water Cherenkov tanks, forming the Surface Detector (SD) with an aperture of $7000 \mathrm{~km}^{2} \mathrm{sr}$ and the 24 peripheral fluorescence telescopes, forming the Fluorescence Detector (FD). 


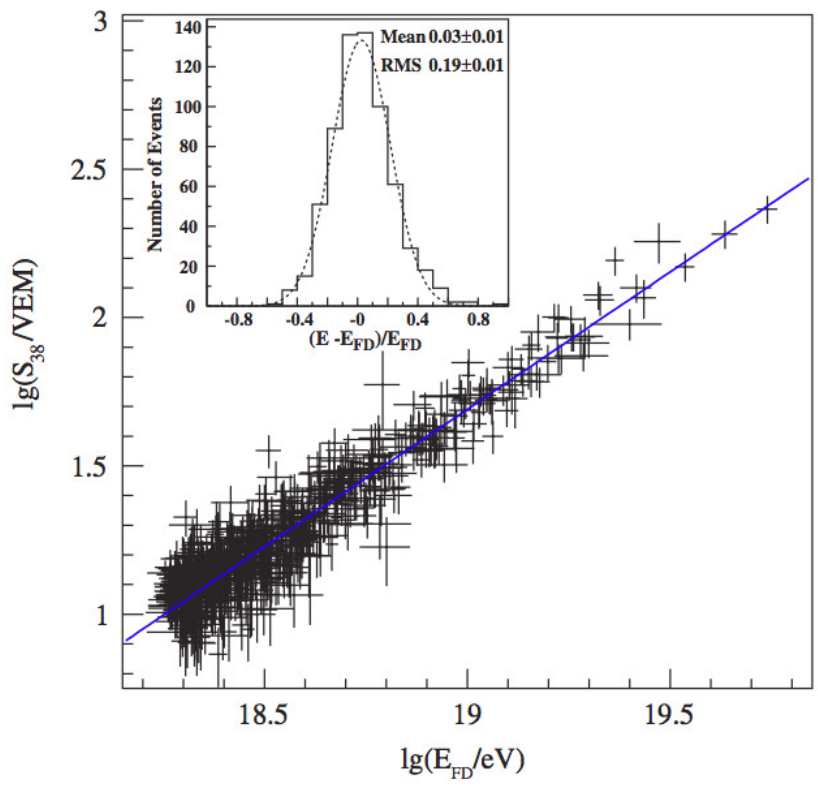

Fig. 2. Correlation between $\log S_{38}$ and $\log E_{F D}$ for a given golden hybrid data set used in the fit. The full line is the best fit for the data. The distribution of the fractional differences between the two energy estimators is shown in the inset. The signal intensity $S_{38}$ is expressed in Vertical Equivalent Muons (VEM) where 1 VEM is the intensity corresponding to one muon crossing the detector with zenith angle equal to zero.

Each water Cherenkov detector (Allekotte et al., 2008) of the surface array is a plastic tank of $10 \mathrm{~m}^{2}$ area and $1.2 \mathrm{~m}$ height hosting 120001 of ultra purified water contained in a Tyvek reflective liner. The Cherenkov light generated by charged particles of the shower crossing the tank is registered by three 9" photomultiplier tubes. Each detector operates autonomously with its own front-end electronics and communication system. A solar energy unit and batteries provide the required $10 \mathrm{~W}$ electrical power. The timing is obtained by a GPS unit and the communications achieved via a wireless system.

Each fluorescence telescope (Abraham et al., 2010b) records the fluorescence light isotropically emitted by the nitrogen molecules of the atmosphere excited by the charged particles during the shower development in air. It consists of a Schmidt optical system with a circular diaphragm, positioned at the centre of curvature of a spherical mirror, that defines the aperture of the system. UV transmitting filters are installed in the entrance aperture and just inside it a ring of corrector lenses is placed to increase the effective aperture by about a factor 2 while preserving the quality of the optical aberrations. The light is focused by a large spherical mirror onto a camera of 440 phototubes camera which allows the $30^{\circ}$ azimuth $\times 28.6^{\circ}$ elevation field of view.

UHECRs are detected measuring two specific features of the particle shower produced in the atmosphere by the in- teraction of the primary particle with air: i) the number of charged particles versus atmospheric depth or longitudinal development and, specifically, the atmospheric depth $\left(X_{\max }\right)$ at which the development reaches its maximum and ii) the signal intensity versus distance from the shower axis (core) sampled with a given surface detector at the detection level at ground or lateral profile.

\section{The hybrid technique}

The joined use of two different techniques allows unprecedented accuracy in reconstructing events from UHECRs. The SD measures the two-dimensional lateral profile of the shower at detection level, that is the number of particles in the shower as a function of their distance from the shower axis at ground, whereas the FD records the longitudinal profile of the shower, that is the number of charged particles during the shower development through the atmosphere as a function of atmospheric depth. The two techniques are complementary allowing a three dimentional reconstruction of the event and also provide a cross-check and redundancy in the measurement of air-shower parameters. The bulk of data are provided by the SD having a $100 \%$ duty-cycle while the FD feeds a minor but very valuable sample of very well reconstructed data having a $16 \%$ duty-cycle as it can only work in clear moonless nights. A subset of doubly reconstructed events, named golden hybrids, are reconstructed simultaneously by both devices and used to calibrate the SD. The subset of golden events used to calibrate the SD events do coincide with the sample of events collected with the FD apart from the necessary cuts for the final event selection.

The Lateral Distribution Function fit of the lateral profile (Rao and Srekanton, 1998) provides a parameter called $S(1000)$, the signal intensity at $1000 \mathrm{~m}$ from the shower axis. $S(1000)$ is a good estimator for the cosmic ray energy. $S(1000)$ depends on the incoming angle because of the shower attenuation in the atmosphere. The variation of $S(1000)$ with the zenith angle has been derived using the so-called constant intensity cut (CIC) method (Hersilet al., 1961). For any shower the $S_{38}=S(1000) / C I C(\theta)$ value, $S(1000)$ normalized at the median of the angle distribution $\theta=38^{\circ}$, represents the new energy estimator. It is the $S(1000)$ value that we would have measured in the case the shower arrived at $38^{\circ}$ zenith angle. A detailed discussion of the way the quantity $S_{38}$ is obtained can be found in Abraham et al. (2008b). The integration of the Gaisser-Hillas curve (Gaisser and Hillas, 1977) adopted to fit the FD longitudinal profile allows a quasi-calorimetric measurement of the energy $E_{F D}$ of the shower to be obtained. Using information from the FD, the energy corresponding to each $S_{38}$ can be estimated almost entirely from data without relying on simulations but only on experimental data, except for the assumption about the missing energy (Abraham et al., 2008b). The correlation of $S_{38}$ with $E_{F D}$ is shown in Fig. 2 together with 
the least-squares fit of the data to a power law $E_{F D}=a S_{38}^{b}$ . The energy resolution, estimated from the fractional difference between $E_{F D}$ and the derived SD energy, is shown like r.m.s. deviation of the distribution. Statistical uncertainties in $S_{38}$ and $E_{F D}$ were assigned to each event.

\section{End to the cosmic ray spectrum?}

With respect to Greisen's persisting question from 1966: "End to the Cosmic Ray Spectrum?" (Greisen, 1966), the Pierre Auger Collaboration today is able to make the following claims: the flux is strongly suppressed above $4 \times 10^{19} \mathrm{eV}$; a single power law hypothesis is rejected with significance $>20 \sigma$; a break in the power law (ankle) has been observed at $\log _{10}(E / e V)=18.61 \pm 0.01$ (Abraham et al., 2008b, 2010c). We have measured the cosmic ray flux with the PAO by applying two different techniques: collecting data with the FD only (that also triggered at least one of the station having the largest signal) and analysing a data sample from SD only, calibrated according the method described in Sect. 3 to extend measurements to lower energies. The energy spectrum of hybrid events is determined from data between November 2005 and May 2008, the spectrum obtained with the SD updates data until the end of December 2008: more than 35000 events fulfill the selection criteria with an actual exposure of $12790 \mathrm{~km}^{2} \mathrm{sr} y \mathrm{r}$. The fluxes obtained with the hybrid events and from the SD array are in good agreement in the overlapping energy range. The dominant systematic uncertainty stems from the overall energy scale, estimated to be $22 \%$. Additional details on the estimation of the systematic uncertainties can be found in Abraham et al. (2008b). Systematic uncertainty on the energy scale is roughly constant in the energy range of Fig. 3. A combined spectrum has been derived with high statistics covering the energy range from $10^{18} \mathrm{eV}$ to above $10^{20} \mathrm{eV}$ using a maximum likelihood method. Since the SD energy estimator is calibrated with the hybrid events, the two spectra have the same systematic uncertainties in the energy scale. The final systematic uncertainty is due to many different contributions. Among them the most important ones are those coming from absolute calibration procedure and from fluorescence yield. The position of the ankle at $\log _{10}(E / e V)=18.61 \pm 0.01$ has been determined by fitting the flux with a broken power law $E^{-\gamma}$. An index of $\gamma=3.26 \pm 0.04$ is found below the ankle. Above the ankle the spectrum follows a power law with index of $\gamma=2.59 \pm 0.02$. A continuation of the power law above the ankle to the highest energies can be rejected with more $20 \sigma$. The suppression is similar to what is expected from the GZK effect (Greisen, 1966; Zatsepin and Kuzmin, 1966) for protons and heavy nuclei as Fe. The combined energy spectrum scaled with $E^{3}$ is shown in comparison with the spectrum obtained with stereo measurements of the HiRes experiment (HiRes Coll., 2010) in Fig. 3. An energy shift within the current systematic uncertainties of the energy scale applied

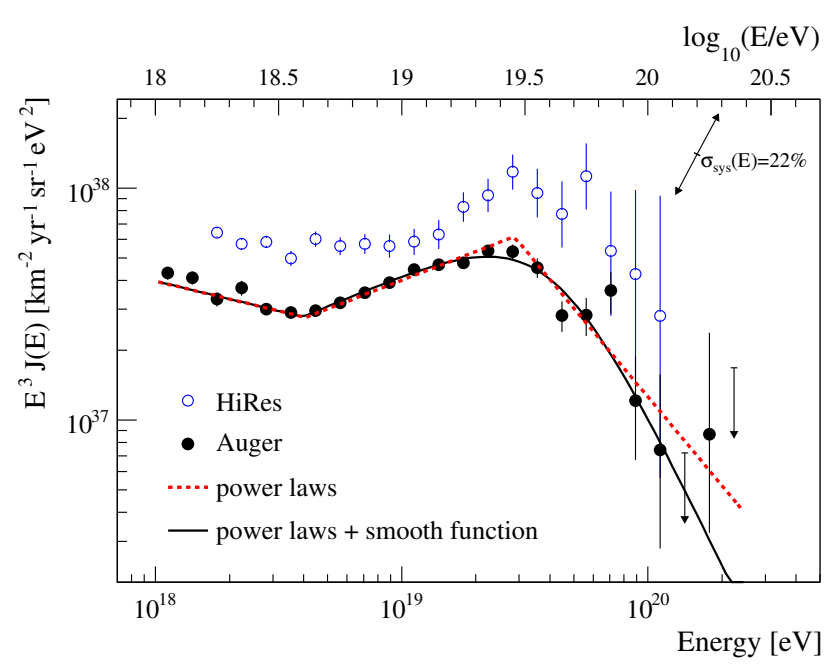

Fig. 3. The combined energy spectrum scaled by $E^{3}$ is fitted with two functions (see Sect. 4) and compared to data from the HiRes experiment. The systematic errors are indicated by arrows, the uncertainty of the energy scale is $22 \%$.

to one or both experiments could account for most differences between them. The ankle seems to be somewhat more sharply defined in our data. This is possibly due to a systematic energy offset between the two experiments. The energy shift is of the order $25 \%$, thus comparable with the average systematic uncertainty in the considered energy range. The characteristic features of the combined spectrum are quantified in two ways: we used three power laws with free breaks between them and a second characterisation is performed by using two power laws in the ankle region and a smoothly changing function at higher energies. Details on the smooth function used for the fit of the energy spectrum can be found in Abraham et al. (2010c). .

\section{What is the composition of the UHECRs?}

To the question:"What are UHECR particles", the current results of the Pierre Auger Collaboration do not answer fully but open new puzzling questions (Abraham et al., 2010d). In fact we observed a change in $X_{\max }$, the atmospheric depth at which the shower reaches its maximum, as a function of the energy around the ankle between $10^{18} \mathrm{eV}$ to above $10^{19} \mathrm{eV}$ where a measurement of the mass composition is crucial to confirm the transition from galactic to extra-galactic cosmic rays predicted by Bottom-Up scenarios and a gradual increase in the mass towards a heavier composition at higher energies.

Assuming the generalisation of Heitler's model (Heitler, 1954) of $e^{-}-\gamma$ cascades to hadron-induced showers and the superposition assumption (Gaisser, 1992), at a given energy, the average of $X_{\max }$, denoted by $<X_{\max }>$, and the width of the $X_{\max }$ distribution, denoted by $R M S\left(X_{\max }\right)$, are both 

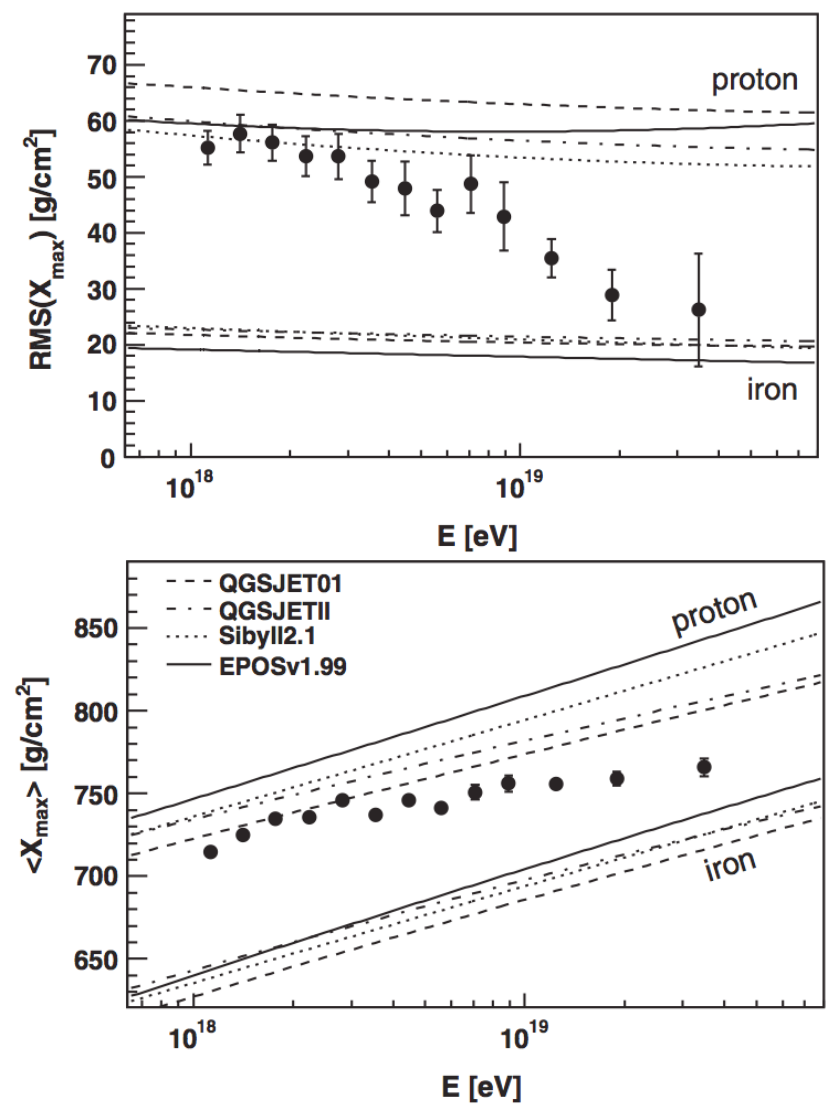

Fig. 4. $<X_{\max }>$ and $R M S\left(X_{\max }\right)$ as a function of energy compared with different air shower simulations .

correlated with the cosmic ray composition. Proton showers penetrate deeper into the atmosphere (larger values of $X_{\max }$ ) and have wider $X_{\max }$ distribution than heavy nuclei. After all cuts over a hybrid data sample from December 2004 to March 2009, 3574 events are selected for the $<X_{\max }>$ analyses. The $<X_{\max }>$ resolution as a function of energy for these data sample is estimated using a detailed simulation of the FD, its calibration and the atmospheric conditions. Reconstruction and event selection give rise to systematic uncertainties of $\leq 13 \mathrm{~g} \mathrm{~cm}^{-2}$ for $<X_{\max }>$ and $\leq 6 \mathrm{~g} \mathrm{~cm}^{-2}$ for the $R M S\left(X_{\max }\right)$. The results were found to be independent of the zenith angle, time periods and FD stations. Our results are reported in Fig. 4. A fit with a constant elongation rate (the so-called change of $<X_{\max }>$ per decade of energy) does not describe our data but using two slopes yields a satisfactory fit. Assuming that the properties of the hadronic interactions do not change significantly over less than two orders of magnitude, this change in the elongation rate, this change in the elongation rate would imply a change in the energy dependence of the composition around the ankle supporting the hypothesis of a transition from galactic to extragalactic cosmic rays in this region. In the rms plot we observe a decrease in fluctuations with energy from about 55 to

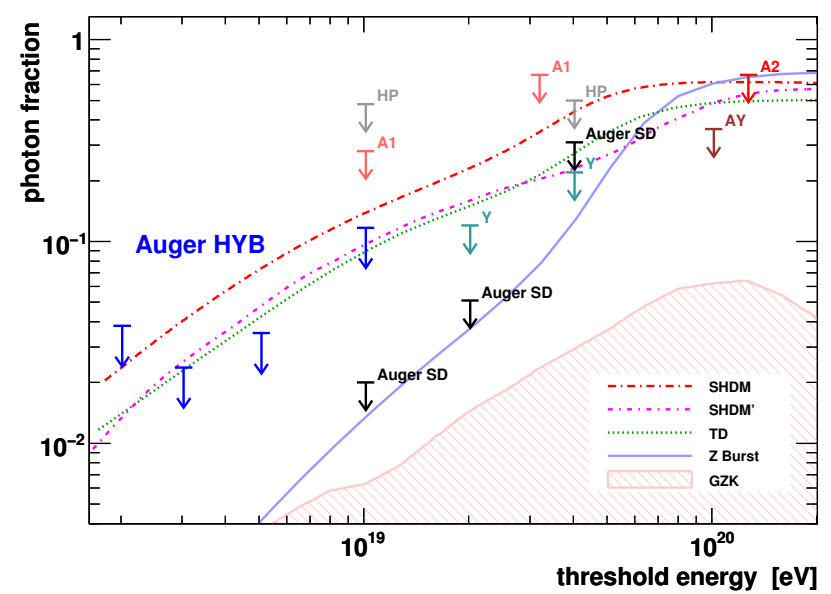

Fig. 5. The black arrows represent the upper limits on the photon flux from the Auger SD above 10, 20, $40 \mathrm{EeV}$ and the blue arrows the upper limits above $2,3,5,10 \mathrm{EeV}$ derived from the FD in comparison with the upper limits on the photon fraction in the integral cosmic-ray flux for different experiments: AGASA (A1,A2), AGASA-Yakutsk (AY), Haverah Park (HP) and Yakutsk (Y). The shaded region shows the expected GZK photon fraction. Lines indicates prediction from Top-Down models .

$26 \mathrm{~g} \mathrm{~cm}^{-2}$ as the energy increases. The decreasing fluctuations are an independent signature of an increasing average mass of the primary particles. For the interpretation of the absolute values fo $X_{\max }$ and $R M S\left(X_{\max }\right)$ a comparison to air shower simulations is needed. As can be seen in Fig.4, here are considerable differences between the results of calculations using different hadronic interaction models. These differences are not necessarily exhaustive since the hadronic interaction models do not cover the full range of possible extrapolations of ow energy acceleration data. If, however, these models provide a realistic description of hadronic interactions at ultrahigh energies. the comparison of data and simulations leads to the same conclusions as above, namely, a gradual increase of the avarage mass of cosmic rays with energy up to $59 \mathrm{EeV}$

\section{Are there ultra high energy photons in our data?}

Could ultra high energy primary particles be photons? The question is pertinent because a substantial photon component is predicted at high energies by non acceleration models. So far we do not find $\gamma$ in our data. The Pierre Auger Collaboration has provided the first upper limit measured with the FD technique (Abraham et al., 2007c), improved with time the SD results (Abraham et al., 2008c) and strongly constraining all Top-Down models (Abraham et al., 2009). At energies above a few $\mathrm{EeV}$, our high accuracy $\left(\approx 20 \mathrm{~g} / \mathrm{cm}^{2}\right)$ measurements of $<X_{\max }>$ provide good discrimination between photons and charged particles. High energy photon showers penetrate deeper in the atmosphere (larger $<X_{\max }>$ ) than 


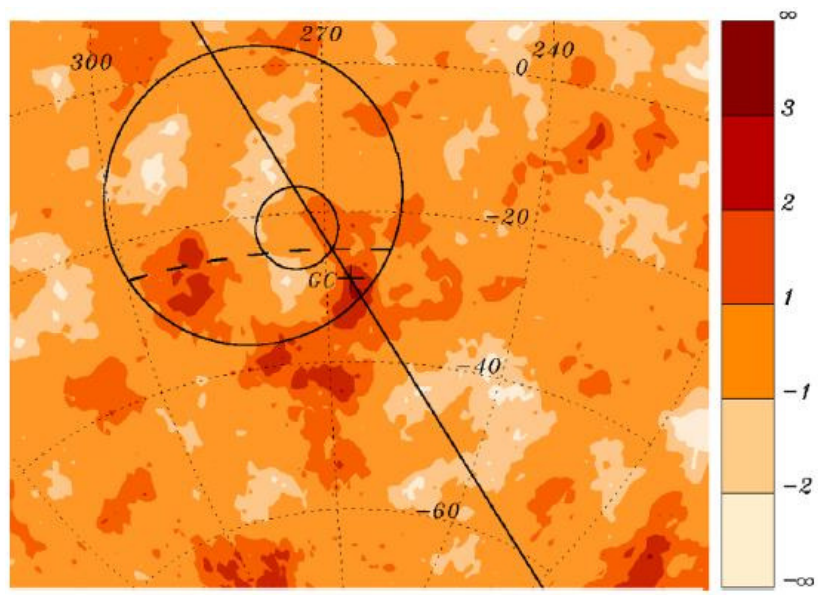

Fig. 6. Map of Cosmic Ray overdensity significances (see text) near the Galactic Centre: the galactic plane is a solid line, the galactic centre is indicated with a cross, the AGASA signal region is the larger circle where the dashed line indicates the field of view boundary of AGASA array..

hadronic showers do but with far fewer muons. This translates by the SD's point of view in a smaller height along the shower axis with respect of hadron-induced shower that develop higher in the atmosphere. Signal risetime and radius of curvature in the SD events are observables sensitive to $\gamma$ induced showers.

We searched for photons using both SD and FD data sets. Above $10^{19} \mathrm{eV}$ no photon candidates were found after applying quality and fiducial cuts, as well as equal acceptance requirements for hadron and photons, from which a photonfraction upper limit was derived. In Fig. 5 the upper limits on the photon fraction in the integral cosmic ray flux for different experiments versus predictions of Top-Down scenarios are shown.

\section{Are there ultra high energy neutrinos in our data?}

Both in conventional acceleration models and in Top-Down scenarios, pions decay to produce an electron to muon neutrino flavor ratio $\left(v_{e}: v_{\mu}\right)$ of order $1: 2$ while $\tau$ neutrinos are heavily suppressed at production. After travelling cosmological distances approximately equal fluxes for each flavor are expected. The idea is to detect $v_{\tau}$-induced events through the emerging $\tau$ produced by neutrinos that enter the Earth just below the horizon (the so-called Earth-skimming neutrinos). We didn't find any candidate for neutrino-induced showers in data recorded up to the present and we established an upper limit on diffuse flux of tau neutrinos in the cosmic radiation (O. Deligny, this Conference, Deligny et al., 2010).

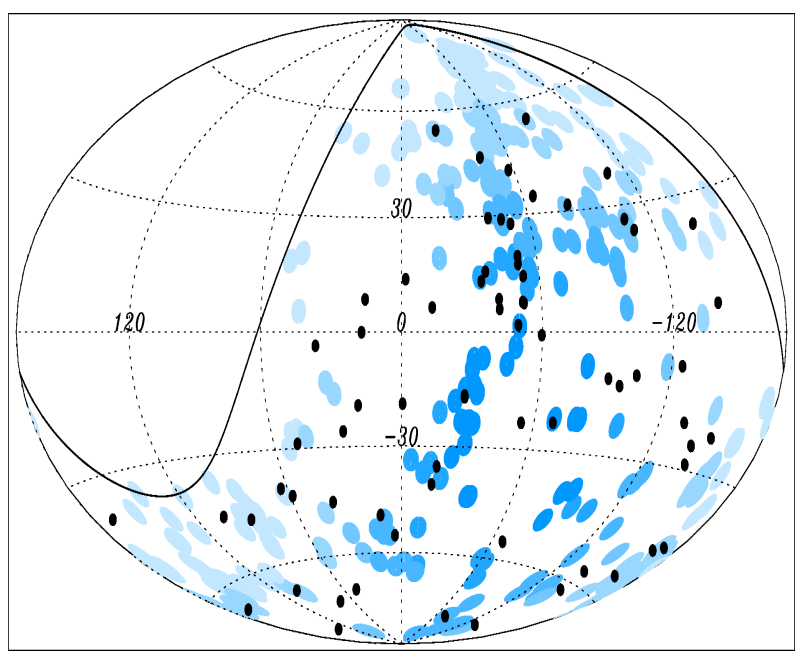

Fig. 7. The 69 arrival directions of CRs with energy larger $55 \mathrm{EeV}$ detected by the Pierre Auger Observatory up to 31 December 2009 (black dots) in an Aitoff-Hammer projection of the sky in galactic coordinates. The positions of 318 AGNs (within $75 \mathrm{Mpc}$ ) in the VCV catalog are reported as circles of radius $3.1^{\circ}$. The solid line represents the field of view of the Southern Observatory for zenith angles smaller than $60^{\circ}$. The exposure-weighted fraction of the sky covered by the AGN circles is $21 \%$.

\section{Where do UHECRs come from?}

If the flux indeed shows a GZK feature at higher energies this implies that the sources of UHECRs must be within our cosmological neighbourhood at distances smaller than $100 \mathrm{Mpc}$. Might this proximity open a window on the nearby Universe allowing an astronomy based on charged particles and the identification of their natural accelerators? In this connection, observation of an excess from the region of the Galactic Center (GC) at the level of $4.5 \sigma$ was reported by AGASA (Teshima et al., 2001). The PAO is suitable for these studies because the GC lies well in the field of view of the experiment (Abraham et al., 2007a). In Fig. 6 we show a map of the $\mathrm{GC}$ region depicting the $\mathrm{Li}-\mathrm{Ma}$ significances $(\mathrm{Li}$ and Ma, 1983) of overdensities in circular windows of $5^{\circ}$ radius, for the SD data with energies in the range $10^{17.9} \div 10^{18.5} \mathrm{eV}$. In our data there is no indication of a statistically significant excess: in the region $1.0<E<2.5 \mathrm{EeV}$ with angular scale of $20^{\circ}$ (same parameters of AGASA), Auger observes 2166 events compared to 2160 expected for flat distribution.

On the contrary, the Pierre Auger Collaboration has reported a correlation of the arrival directions of ultra high energy cosmic rays with energies larger than $56 \mathrm{EeV}$ and the position of the nearby objects from the Véron-Cetty and Véron (VCV) catalog (Véron-Cetty and Véron, 2006) of quasars and Active Galactic Nuclei (AGN) (Abraham et al., 2007b, 2008a). The null hypothesis was rejected with 99\% confidence level based on a single-trial test motivated by early data and confirmed by the data collected subse- 


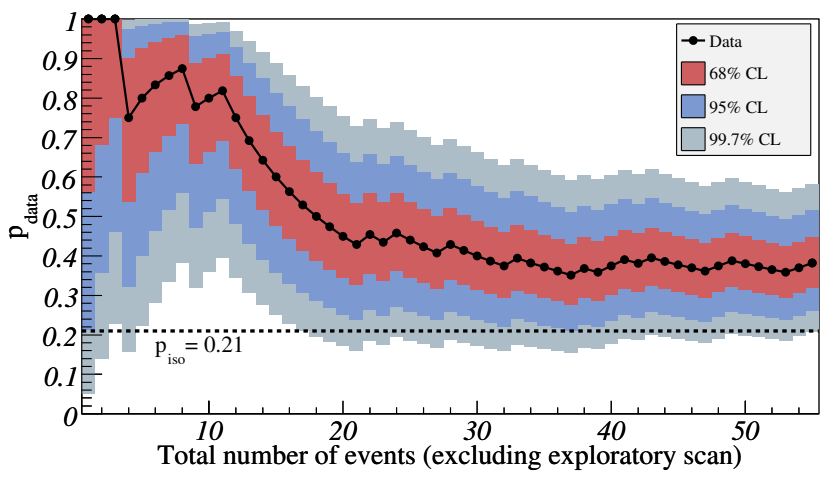

Fig. 8. The most likely value of the degree of correlation $p_{d a t a}=$ $k / N$ (black dots) plotted as function of the time-ordered events excluding the events of the initial exploratory scan (see Sect. 8). The $68 \%, 95 \%$ and $99.7 \%$ regions around the dots are shown. The horizontal dashed line shows the p-value for the isotropic expectation

quently to the definition of the test. An update of this analysis has been recently published including events detected up to 31 December 2009 (Abraham et al., 2010a). The integrated exposure is $20370 \mathrm{~km}^{2}$ sr year. This event sample recorded with the SD between 1 January 2004 and 31 December 2009 with zenith angle $\theta<60^{\circ}$ and reconstructed energies $E \geq 56 \mathrm{EeV}$ (with exactly the same reconstruction algorithm, energy calibration and quality cuts for event selection as in the exploratory scan) is made of 69 arrival directions. The same conditions for the comparison were applied: the angular distance from the AGNs is still $3.1^{\circ}$, as in the exploratory scan. Only AGNs in the VCV catalog with redshift $z \leq 0.018$ were considered. The arrival directions of the 69 primary particles are reported in Fig. 7 and compared with the position in galactic coordinates of 318 AGNs from VCV catalog. The exposure-weighted fraction of the sky covered by the $3.1^{\circ}$ angular regions around the AGNs is $21 \%$. In other words, this is the fraction of events that one expects to correlate under the isotropic hypothesis. The current status is therefore better seen in Fig. 8 where the most likely value of the degree of correlation $p_{\text {data }}=k / N$ (black dots), where $k$ is the number correlating with objects in the VCV catalog and $N$ is the total number of events (Abraham et al., 2010a), is plotted as function of the time-ordered events excluding the events of the initial exploratory scan. The $68 \%, 95 \%$ and 99.7\% regions around the dots are also shown. The horizontal dashed line shows the p-value for the isotropic expectation. The amount of correlation observed decreased from $69 \%$ with $9 / 13$ to its current estimate of $38 \%$ based on 21 correlations out of 55 events. We note that 9 of 55 events are within $10^{\circ}$ of the galactic plane and none of them correlates within $3.1^{\circ}$ with the astronomical objects under consideration. Incompletness of the VCV catalog due to obscuration by the Milky Way or larger magnetic fields along galactic disk are potential causes. We have therefore examined cross- correlation between arrival directions of UHECRs and positions of the objects in the 2MRS (Jarrett et al., 2000) and Swift-BAT (Tueller et al., 2010) catalogs. Only astrophysical objects within $200 \mathrm{Mpc}$ were considered and in the case of the 2MRS catalog a region $\left( \pm 10^{\circ}\right)$ around the galactic plane was excluded, due to incompleteness of the 2MRS catalog in the galactic plane region. We have observed correlations in excess of isotropic expectations in all considered cases. Additional details can be found in Abraham et al. (2010a).

\section{Conclusions}

The Pierre Auger Observatory was completed in 2008 and it is currently taking data. It is the first experiment in high energy cosmic ray physics using the powerful hybrid technique: joined use of fluorescence telescopes and surface stations to achieve high performances and unprecedented accuracy. Until now, about 6 years of data (million cosmic ray events) have been acquired and analysed. Unique results on the all particle energy spectrum, mass composition, anisotropy on small and large scale, photon and tau neutrino upper limits have been produced. Outstanding steps toward a complete understanding of the nature of ultra high energy cosmic rays have been taken but no exhaustive answers given. The current results open intriguing questions that only a large amount of additional data, coming in the next years of operation of the Observatory, can disentangle.

Edited by: K. Scherer

Reviewed by: I. A. Grenier and another anonymous referee

\section{References}

Abbasi, R. U. et al. (HiRes Collaboration): Measurement of the Flux of Ultra High Energy Cosmic Rays by the Stereo Technique, Astropart. Phys., 32, 53-60, 2009.

Abraham, J. et al. (Pierre Auger Collaboration): Properties and performance of the prototype instrument for the Pierre Auger Observatory, Nucl. Instrum. Meth. A, 523, 50-95, 2004.

Abraham, J. et al. (Pierre Auger Collaboration): Anisotropy studies around the galactic centre at EeV energies with the Auger Observatory, Astropart. Phys., 27, 244-253, 2007a.

Abraham, J. et al. (Pierre Auger Collaboration): Correlation of the Ultra-Highest-Energy Cosmic Rays with Nearby Extragalactic Objects, Science, 318, 938-943, 2007b.

Abraham, J. et al. (Pierre Auger Collaboration): An upper limit to the photon fraction in cosmic rays above $10^{19} \mathrm{eV}$ from the Pierre Auger Observatory, Astropart. Phys., 27, 155-168, 2007c.

Abraham, J. et al. (Pierre Auger Collaboration): Correlation of the highest-energy cosmic rays with the positions of nearby active galactic nuclei, Astropart. Phys., 29, 188-204, 2008a.

Abraham, J. et al. (Pierre Auger Collaboration): Observation of the Suppression of the Flux of Cosmic Rays above $4 \times 10^{19} \mathrm{eV}$, Phys. Rev. Lett., 101, 061101, 2008b.

Abraham, J. et al. (Pierre Auger Collaboration): Upper limit on the cosmic-ray photon flux above $10^{19} \mathrm{eV}$ using the surface detector 
of the Pierre Auger Observatory, Astropart. Phys., 29, 243-256, 2008c.

Abraham, J. et al. (Pierre Auger Collaboration): Upper limit on the cosmic-ray photon fraction at EeV energies from the Pierre Auger Observatory, Astropart. Phys., 31, 399-406, 2009.

Abraham, J. et al. (Pierre Auger Collaboration): Update on the correlation of the highest energy cosmic rays with nearby extragalactic matter, Astropart. Phys., 34, 314-326, 2010a.

Abraham, J. et al. (Pierre Auger Collaboration):The fluorescence detector of the Pierre Auger Observatory, Nucl. Instrum. Meth. A, 620, 227-251, 2010b.

Abraham, J. et al. (Pierre Auger Collaboration): Measurement of the energy spectrum of cosmic rays above $10^{18} \mathrm{eV}$ using the Pierre Auger Observatory, Phys. Lett. B, 685, 239-246, 2010.

Abraham, J. et al. (Pierre Auger Collaboration): Measurement of the Depth of Maximum of Extensive Air Showers above $10^{18} \mathrm{eV}$, Phys. Rev. Lett., 104, 091101, 2010d.

Allekotte, I., Barbosa, A., Bauleo, P. et al.: The Surface Detector System of the Pierre Auger Observatory, Nucl. Instrum. Meth. A, 586, 409-420, 2008.

Deligny, O. et al. (Pierre Auger Collaboration): Limits on the diffuse flux of ultra high energy neutrinos using the Pierre Auger Observatory, in: Proc. 22nd ECRS, Turku, Finland, 2010.

Gaisser, T. K.: Cosmic Rays and Particle Physics, Cambridge Univ. Press, New York, 1992.

Gaisser, T. K. and Hillas, A. M.: Reliability of the method of constant intensity cuts for reconstructing the average development of vertical showers, in Proceedings of the 15th ICRC, Plovdiv, Bulgaria, 8, 353, 1977.
Greisen, K.: End to the Cosmic-Ray Spectrum?, Phys. Rev. Lett., 17, 748-750, 1966.

Heitler, W.: The Quantum Theory of Radiation, Oxford Univ. Press, New York, 1954.

Hersil, J., Escobar, I., Scott, D. et al.: Observations of Extensive Air Showers near the Maximum of Their Longitudinal Development, Phys. Rev. Lett., 6, 22-23, 1961.

Jarrett, T. H., Chester, T., Cutri, R. et al.: 2Mass Extended Source Catalog: Overview and Algorithms, Astron. J., 119, 2498-2531, 2000.

Li, T. P. and Ma, Y. Q.: Analysis methods for results in gamma-ray astronomy, Astrophys. J., 272, 317, 1983.

Linsley, J.: Evidence for a Primary Cosmic-Ray Particle with Energy $10^{20} \mathrm{eV}$, Phys. Rev. Lett., 10, 146148, 1963.

Nagano, M. and Watson, A.: Observations and implications of the ultrahigh-energy cosmic rays, Rev. Mod. Phys., 72, 689-732, 2000.

Rao, M. V. S. and Sreekantan, B. V.: Extensive Air Shower, World Scient. Publ. Comp., Singapore, 1998.

Teshima, M., Chikawa, M., Fukushima, M. et al.: Anisotropy of cosmic-ray arrival directions at $10^{18} \mathrm{eV}$ observed by AGASA, in: Proc. 27th ICRC, Hamburg, Germany, 1, 337, 2001.

Tueller, J., Baumgartner, W. H., Markwardt, C. B. et al.: The 22Month Swift-BAT All-Sky Hard X-ray xSurvey, Astrophys. J. Suppl., 186, 378-405, 2010.

Véron-Cetty, M.-P. and Véron, P.: A catalogue of quasars and active nuclei: 12th edition, Astron. Astrophys., 455, 773-777, 2006.

Zatsepin, G. T. and Kuzmin, V. A.: Upper Limit of the Spectrum of Cosmic Rays, Sov. Phys. JETP Lett., 4, 78, 1966. 